\title{
ANÁLISE DOS MODELOS DE TURBULÊNCIA $k-\varepsilon, k-\omega$ E SST NO ESCOAMENTO CRUZADO SOBRE UM CILINDRO
}

\author{
Analysis of the sst and $k-\varepsilon, k-\omega$ turbulence models in cross flow on a cylinder \\ Chrystian Martins Freitas ${ }^{1}$; Anderson José Antonietti ${ }^{2}$ \\ ${ }^{1}$ Engenheiro Mecânico formado pela Universidade do Extremo Sul Catarinense - UNESC, Criciúma, \\ SC.E-mail: chrystianmfreitas@gmail.com \\ ${ }^{2}$ Prof. Me. do Instituto Federal de Santa Catarina - IFSC, Jaraguá do Sul - Rau, SC. E-mail: anderson. \\ jose@ifsc.edu.br
}

Data do recebimento: 07/01/2020 - Data do aceite: 09/04/2020

\begin{abstract}
RESUMO: Este trabalho apresenta os resultados do estudo do escoamento cruzado em um cilindro liso, por meio de simulações computacionais, utilizando os modelos de turbulência $k$ - $\varepsilon, k-\omega$ e SST. A metodologia empregada para o estudo considerou os resultados do coeficiente de arrasto presentes na literatura como critério de validação para os dados obtidos nas simulações. Com o objetivo de apontar qual o modelo de turbulência apresenta melhor capacidade preditiva para este de tipo geometria, consideraram-se três modelos dentre os disponíveis para uso no software Ansys CFX, aplicando-os nas condições-padrão do software. Os resultados obtidos mostraram que o modelo $k$ - $\omega$ é o mais indicado para toda a faixa de Reynolds analisada no escoamento cruzado em cilindro liso, pois, além de obter bons resultados, esse modelo também demanda um menor tempo computacional para a simulação, em comparação com o modelo SST.
\end{abstract}

Palavras-chave: Simulação. Mecânica dos Fluidos. Força de Arrasto.

\begin{abstract}
This work presents the results of the study of cross flow on a smooth cylinder through computational simulations using the $k-\varepsilon, k-\omega$ and SST turbulence models. The methodology used for the study considered the results of the drag coefficient present in the literature as validation criterion for the data obtained in the simulations. In order to indicate which turbulence
\end{abstract}


model has the best predictive capacity for this type of geometry, three models were considered among those available for use in the Ansys CFX software, applying them under the standard software conditions. The obtained results showed that the $k-\omega$ model is the most suitable for the entire Reynolds band analyzed on the cross-flow in a smooth cylinder, because in addition to obtaining good results this model also demands a smaller computational time for the simulation compared to the SST model.

Keywords: Simulation. Fluid Mechanics. Drag Force.

\section{Introdução}

A aerodinâmica é a área responsável pelo estudo do fluxo de ar e das forças exercidas por ele sobre o objeto de estudo. Esse estudo é imprescindível para melhorar o desempenho, consumo e estabilidade, seja em carros de passeio, aviões, bolas de futebol, ou qualquer estrutura sujeita ao escoamento de fluido. O estudo aerodinâmico é geralmente realizado em túneis de vento, onde é possível obter, além da visualização do escoamento, as forças resultantes do escoamento sobre o modelo, ou seja, as forças de sustentação e arrasto.

$\mathrm{Na}$ maioria das vezes, quando há impossibilidade de realizar os testes em um túnel de vento, aplicam-se métodos numéricos por meio de softwares CAE (Computer Aided Engineering). Os programas de engenharia assistida por computador (CAE) realizam análise por elementos finitos (FEA), simulação dinâmica (análise de forças), análise fluidodinâmica por volumes finitos (CFD) e outras análises importantes no campo da engenharia. Em específico, o Método dos Volumes Finitos (MVF) é o mais utilizado para problemas envolvendo escoamento de fluidos. Esse método consiste na discretização de um domínio fluido em um número finito de volumes de controle. A cada volume de controle são aplicadas as formas discretizadas das equações de conservação e quantidade de movimento, com o objetivo de se obter um sistema de equações algébricas lineares. Esse sistema é então resolvido iterativamente por um solucionador algébrico.

Dentre as principais dificuldades envolvidas no estudo fluidodinâmico está a escolha correta do modelo de turbulência. O modelo de turbulência empregado em análises que envolvem a modelagem correta da camada-limite fluidodinâmica é de fundamental importância, pois dela dependem diretamente as forças de sustentação e arrasto que agem sobre o modelo. De modo a comprovar essa importância, neste trabalho é desenvolvida uma análise fluidodinâmica computacional de um cilindro em escoamento cruzado, usando diferentes modelos de turbulência disponíveis no software Ansys CFX. Busca-se, portanto, determinar o modelo de turbulência que melhor descreve o escoamento cruzado sobre um cilindro liso na obtenção do coeficiente de arrasto em comparação com dados experimentais apresentados por Fox e McDonald (2014).

\section{Fundamentação Teórica}

A predição dos indicadores de mérito aerodinâmico - coeficientes de sustentação 
e arrasto - é fundamental no projeto dimensional de geometrias expostas ao escoamento externo. Nesse contexto, a mecânica dos fluidos computacional mostra-se uma alternativa atraente, se não a única, muitas vezes, na obtenção ou estimativa desses indicadores.

A maioria dos escoamentos de interesse da engenharia são turbulentos. Mesmo com todo o avanço tecnológico de simulações numéricas, a análise do escoamento turbulento ainda é um grande desafio e, apesar de existirem vários modelos de turbulência para simulação, nenhum deles é capaz de prever com precisão valores para todos os tipos de escoamento. A dificuldade de modelagem da turbulência está ligada à representação do escoamento que ocorre na região interna da camada-limite, onde a intensidade dos gradientes de velocidade, associados aos efeitos da viscosidade e da difusividade turbulenta de transporte de quantidade de movimento, são difíceis de serem tratados (SOARES, 2006). Existe a necessidade de estudos para cada caso e cada tipo de geometria para poder utilizar o modelo de turbulência correto e obter melhores resultados.

Os modelos de turbulência exercem papel fundamental na determinação das condições fluidodinâmicas de várias aplicações na engenharia. Para este trabalho, os modelos de turbulência analisados serão o $k-\varepsilon$, o $k-\omega$ e o SST, sob as condições-padrão apresentadas no software Ansys CFX.

\section{Modelo de Turbulência $k-\varepsilon$}

O modelo de turbulência $k-\varepsilon$, proposto por Harlow e Nakayama (1968), implementado por Jones e Launder (1972) e complementado posteriormente por Launder e Spalding (1974), é um dos modelos mais comuns e mais usados atualmente. Este modelo utiliza duas equações de conservação para determinar a viscosidade turbulenta que permite determinar as tensões de Reynolds, ou seja, resolvidas essas equações pode-se representar as propriedades turbulentas do escoamento. A primeira equação representa a energia cinética de turbulência $k$; a segunda equação é a taxa de dissipação da energia cinética de turbulência $\varepsilon$. O modelo $k$ - $\varepsilon$ possui robustez, economia e razoável precisão para uma série de escoamentos turbulentos (PEREIRA, 2010), sendo mais utilizado para escoamento afastado da parede, em fluxos de camada de cisalhamento livre com gradientes de pressão relativamente pequenos e escoamentos com altos números de Reynolds (BENDER, 2017). No entanto, apresenta problemas em alguns casos como em escoamento não confinado, escoamento rotativo e escoamento em dutos não circulares.

\section{Modelo de Turbulência $k-\omega$}

O modelo $k$ - $\omega$ padrão é baseado no modelo $k$ - $\omega$ proposto inicialmente por Wilcox (1993). Para calcular a viscosidade turbulenta, esse modelo apoia-se na utilização da energia cinética de turbulência $k$ e da taxa de dissipação especifica $\omega$. De modo a conseguir um modelo com maior precisão para avaliar escoamentos cisalhantes livres, foram realizadas algumas modificações no modelo original, adicionando termos nas equações de $k$ e $\omega$. Esse modelo apresenta resultados satisfatórios para escoamento em canais tanto para Reynolds altos quanto baixos, sendo também muito utilizado em casos em que o modelo $k-\varepsilon$ não é exato, como em escoamentos que possuem gradiente de pressão adverso (Figura 1a) e para escoamentos recirculantes (Figura 1b) (SOARES, 2006). 
Figura 1 - Escoamento viscoso em torno de uma esfera (a) e escoamento recirculante (b)

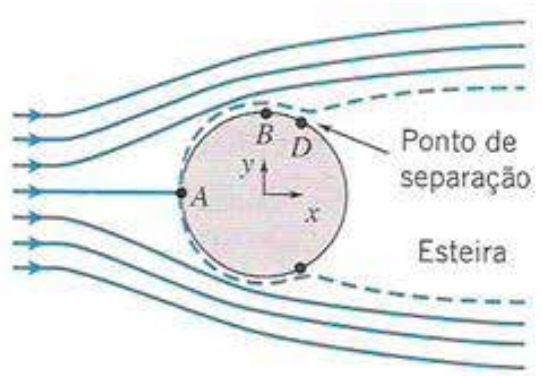

(a)

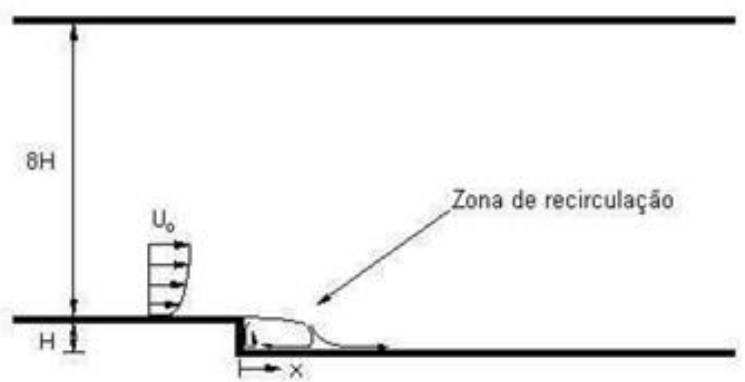

(b)

Fonte: (a) FOX; MCDONALD (2014); (b) SOARES (2006)

\section{Modelo de Turbulência SST - Shear Stress Transport}

O modelo de turbulência SST (Transporte de Tensão Cisalhante), proposto por Menter (1994), é um modelo que combina as vantagens de dois modelos clássicos de turbulência, o modelo $k-\varepsilon$ e o $k$ - $\omega$. É, assim, um modelo com dois conjuntos de equações: na região externa do escoamento usa a equa- ção transformada de transporte do modelo $k-\varepsilon$; e as equações do modelo $k-\omega$ são usadas em regiões próximas às paredes, em que se mostram mais eficazes.

\section{Percurso Metodológico}

Com o intuito de desenvolver um estudo comparativo do desempenho de modelos de turbulência na descrição do escoamento

Figura 2 - Coeficiente de arrasto para um cilindro circular liso em função do número de Reynolds

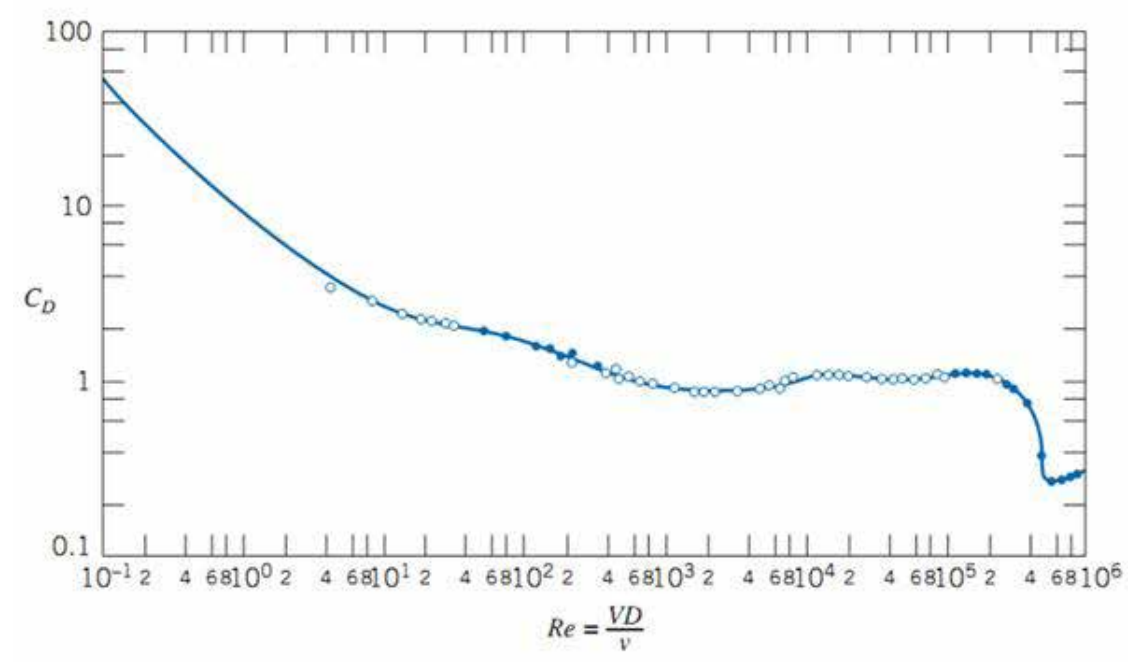

Fonte: FOX; MCDONALD (2014) 
cruzado sobre um cilindro liso, utiliza-se como critério de avaliação o gráfico da Figura 2 , que apresenta valores do coeficiente de arrasto para cilindros em função do número de Reynolds $(R e)$.

A escolha dos pontos para a análise foi baseada nas regiões específicas de desenvolvimento do escoamento, sinalizadas pelas letras A, B, C, D e E, do gráfico da Figura 3.

Por meio da análise de cada um desses pontos (Figura 4), define-se o segmento do gráfico a ser considerado, tendo como base as velocidades que produzem um escoamento turbulento, ou seja, as que resultam em números de Reynolds maiores que $1 \times 10^{2}$, início da esteira oscilante de von Karman (Ponto C), e menores que $7 \times 10^{5}$, que caracteriza a chamada crise de arrasto (Ponto E).
Figura 4 - Estruturas típicas dos escoamentos referentes aos pontos indicados no gráfico

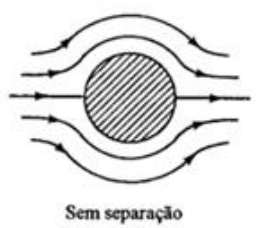

(A)

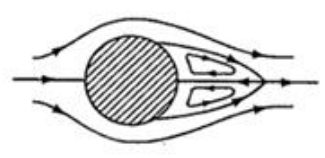

Botha de separaçlo permanente

(B)

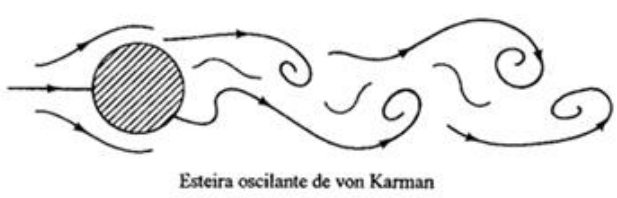

(C)

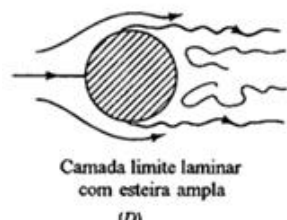

(D)

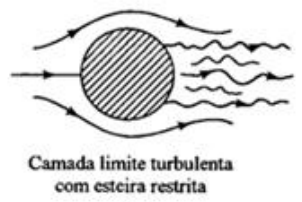

(E)
Fonte: MUNSON; YOUNG; OKIISHI (2004)

Figura 3 - Gráfico do coeficiente de arrasto

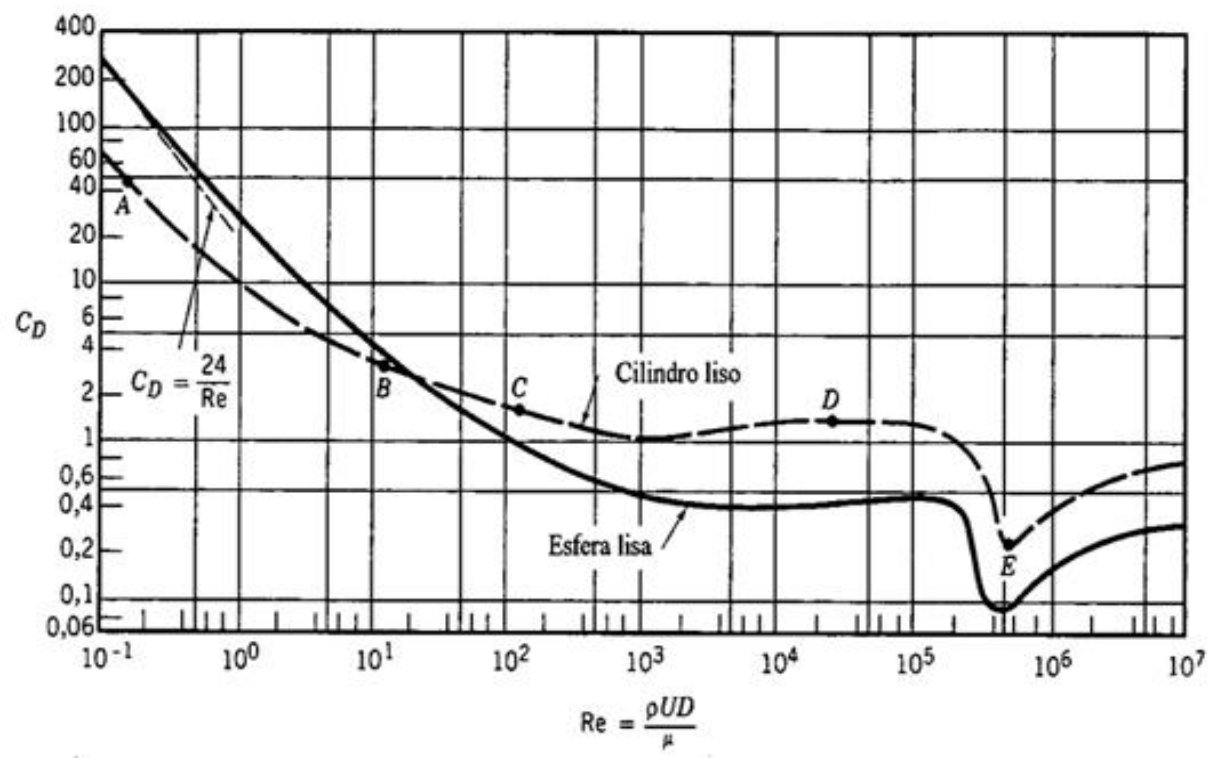

Fonte: MUNSON; YOUNG; OKIISHI (2004) 
Os pontos escolhidos dentro do segmento C-E são apresentados na Tabela I.

Tabela I - Valores de Reynolds e dos coeficientes de arrasto escolhidos para a análise

\begin{tabular}{cc}
\hline$R e$ & $C_{D}{ }^{*}$ \\
\hline 200 & 1,625 \\
4000 & 1 \\
20000 & 1,25 \\
500000 & 0,3 \\
\hline
\end{tabular}

Onde $C_{D}$ * representa o valor do coeficiente de arrasto encontrado na literatura para o respectivo número de Reynolds.

\section{Modelo Computacional}

Os testes foram realizados no software de simulação numérica Ansys, no qual foi criada a geometria de estudo e o domínio computacional, aplicada a malha de volumes finitos, atribuídas as condições de contorno do problema, obtida a solução e analisados os resultados.

A geometria utilizada nos testes é um cilindro de diâmetro de $0,1 \mathrm{~m}$ e espessura de $0,005 \mathrm{~m}$, sendo que o objetivo foi determinar a força de arrasto sofrida durante o escoamento. Para isso, é necessário que o domínio computacional, volume em torno do cilindro por onde o escoamento flui, seja bem dimensionado. O manual do software Ansys CFX sugere que as dimensões do domínio computacional 3D respeitem os limites mínimos apresentados na Figura 5.

Figura 5 - Distâncias recomendadas

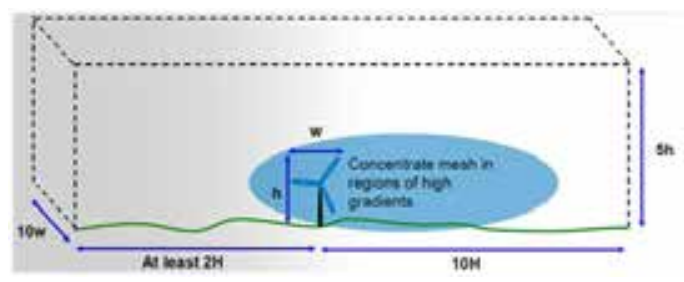

Fonte: Ansys CFX Inc., Solver Theory
Para o caso de estudo, o domínio computacional foi criado em três dimensões, porém o seu comportamento é induzido para um domínio bidimensional, pois as forças na terceira dimensão são pequenas, além de serem desnecessárias para a análise. Isso é possível devido à discretização da espessura do domínio em apenas um volume de controle nessa direção. O domínio computacional, bem como suas dimensões, é apresentado na Figura 6.

Figura 6 - Geometria do domínio computacional

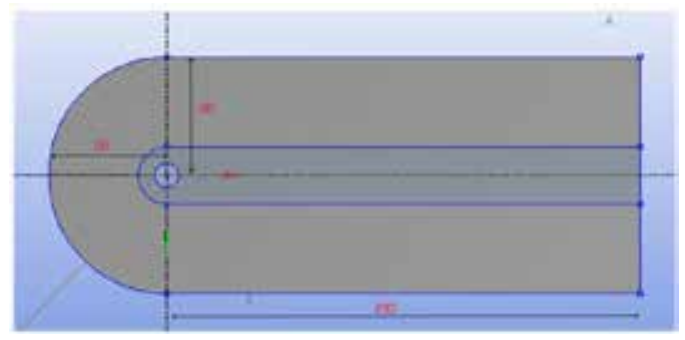

A malha computacional empregada na realização das simulações passou por um processo de avaliação de desempenho em função do refinamento imposto, ou seja, inicialmente foi aplicada uma malha mais simples sobre a geometria, aumentando progressivamente o número de volumes, o que é chamado de refinamento de malha. Esse refinamento foi realizado até que não houve mudanças significativas entre os valores obtidos. Existem dois métodos para realizar esse refino: o método global, que abrange todo o domínio computacional; e o método local, onde é possível especificar apenas a região de interesse. A Tabela II apresenta os testes de independência de malha realizados considerando-se uma determinada velocidade de escoamento.

Com base no teste de independência de malha, foi considerado que a malha $\mathrm{D}$ é a mais adequada para a realização da análise. A Figura 7 apresenta a malha aplicada sobre o domínio fluido. 
Tabela II - Teste de independência de malha

\begin{tabular}{cccc}
\hline Malha & $C_{D}$ & $\mathrm{~N}^{\circ}$ de volumes & $\mathrm{N}^{\circ}$ de nós \\
\hline $\mathrm{A}$ & 0,806735 & 2886 & 5982 \\
$\mathrm{~B}$ & 0,892853 & 14398 & 29182 \\
$\mathrm{C}$ & 1,16791 & 42477 & 85482 \\
$\mathrm{D}$ & 1,16123 & 57705 & 116156 \\
\hline
\end{tabular}

Figura 7 - Malha do domínio computacional

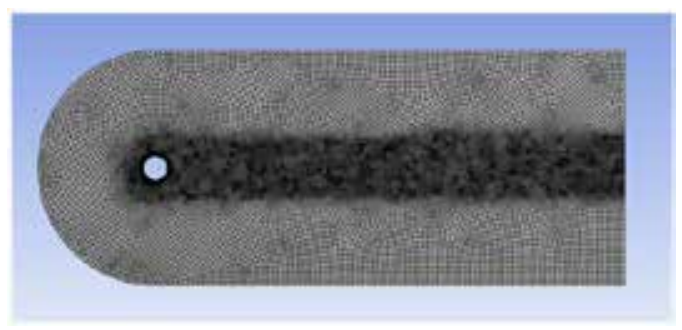

Essa malha computacional foi empregada em todos os testes realizados, alterando-se apenas as condições de contorno para cada caso.

Para a obtenção de bons resultados é de extrema importância definir as condições de contorno corretas a serem utilizadas nas simulações. As condições de contorno representam o conjunto de características de determinado escoamento, como fluido de trabalho, condições de entrada e saída de fluxo, etc. Neste trabalho, optou-se por realizar o escoamento de água com temperatura uniforme de $25^{\circ} \mathrm{C}$ em torno do cilindro, com velocidades de entrada $(V)$ em função do número de Reynolds $(R e)$ relativo a cada ponto escolhido no gráfico, de acordo com a equação

$$
V=\frac{R e \cdot \mu}{\rho . D^{\prime}}
$$

sendo $\mu$ a viscosidade dinâmica do fluido (N.s $\left./ \mathrm{m}^{2}\right), \rho$ a massa específica $\left(\mathrm{kg} / \mathrm{m}^{3}\right)$ e $D$ o diâmetro do cilindro (m). Para os quatro pontos escolhidos têm-se as velocidades mostradas na Tabela III:
Tabela III - Velocidade de entrada em função do número de Reynolds

\begin{tabular}{cc}
\hline$R e$ & $V[\mathrm{~m} / \mathrm{s}]$ \\
\hline 200 & 0,00178 \\
4000 & 0,03572 \\
20000 & 0,17851 \\
500000 & 4,46289 \\
\hline
\end{tabular}

De modo a evitar a interferência das paredes laterais do domínio computacional no escoamento sobre o perfil cilíndrico, aplica-se às mesmas a condição de contorno de simetria.

O critério de convergência adotado foi o RMS - Root Mean Square dos valores residuais, sendo o menor valor igual a $1 \times 10^{-5}$ para todas as equações. $\mathrm{O}$ esquema de interpolação adotado foi o High Resolution.

O objetivo das simulações é obter o coeficiente de arrasto $\left(C_{D}\right)$, para cada modelo de turbulência aplicado, de acordo com a equação

$$
C_{D}=\frac{2 \cdot F_{D}}{\rho V^{2} A}
$$

sendo $F_{D}$ a força de arrasto $(\mathrm{N})$ e $A$ a área projetada do cilindro $\left(\mathrm{m}^{2}\right)$.

Considerando as mesmas condições anteriores em todos os testes, procura-se verificar qual dos três modelos de turbulência $(k-\varepsilon, k-\omega$ ou SST), presentes no software comercial Ansys, melhor se aplica no caso em estudo. Vale ressaltar que os modelos utilizados respeitaram a condição-padrão do software, não sendo realizado nenhum ajuste no modo de aplicação dos mesmos.

\section{Resultados e Discussão}

Nesta seção são apresentados os resultados obtidos para o coeficiente de arrasto nos 
quatro pontos de análise escolhidos, e suas variações quanto ao modelo de turbulência. Todas as simulações foram executadas utilizando a mesma malha e as mesmas condições de contorno.

\section{Resultados para o modelo de turbulência $k-\varepsilon$}

Para o modelo de turbulência $k$ - $\varepsilon$ foram obtidos os resultados apresentados na Tabela IV.

Tabela IV - Resultado do coeficiente de arrasto para o modelo $k-\varepsilon$

\begin{tabular}{ccccc}
\hline$R e$ & $V[\mathrm{~m} / \mathrm{s}]$ & $C_{D}(k-\varepsilon)$ & $C_{D}{ }^{*}$ & Erro $(\%)$ \\
\hline 200 & 0,00178 & 1,49333 & 1,625 & $-8,10$ \\
4000 & 0,03572 & 0,40686 & 1,0 & $-59,31$ \\
20000 & 0,17851 & 0,25862 & 1,25 & $-79,30$ \\
500000 & 4,46289 & 0,54724 & 0,3 & 82,41 \\
\hline
\end{tabular}

A Figura 8 apresenta a localização desses pontos.

Pode-se observar, nesse caso, que o modelo de turbulência $k$ - $\varepsilon$ não se mostra uma boa alternativa para este tipo de escoamento, validando suas características. Sabe-se que este modelo não apresenta bons resultados quando aplicado em escoamentos não confinados, em casos de separação da camada-limite e em camadas-limite curvadas, as quais são características da geometria em estudo.

\section{Resultados para o modelo de turbulência $k-\omega$}

Para o modelo de turbulência $k$ - $\omega$ os resultados obtidos são apresentados na Tabela V.

Tabela V - Resultado do coeficiente de arrasto para o modelo $k-\omega$

\begin{tabular}{ccccc}
\hline $\operatorname{Re}$ & $V[\mathrm{~m} / \mathrm{s}]$ & $C_{D}(k-)$ & $C_{D}{ }^{*}$ & Erro (\%) \\
\hline 200 & 0,00178 & 1,75452 & 1,625 & 7,97 \\
4000 & 0,03572 & 0,95798 & 1,0 & $-4,20$ \\
20000 & 0,17851 & 1,29358 & 1,25 & 3,48 \\
500000 & 4,46289 & 0,33242 & 0,3 & 10,80 \\
\hline
\end{tabular}

A Figura 9 apresenta a localização desses pontos.

Figura 8 - Pontos respectivos aos coeficientes de arrasto obtidos para o modelo $k$ - $\varepsilon$, destacados no gráfico pelo o símbolo $x$

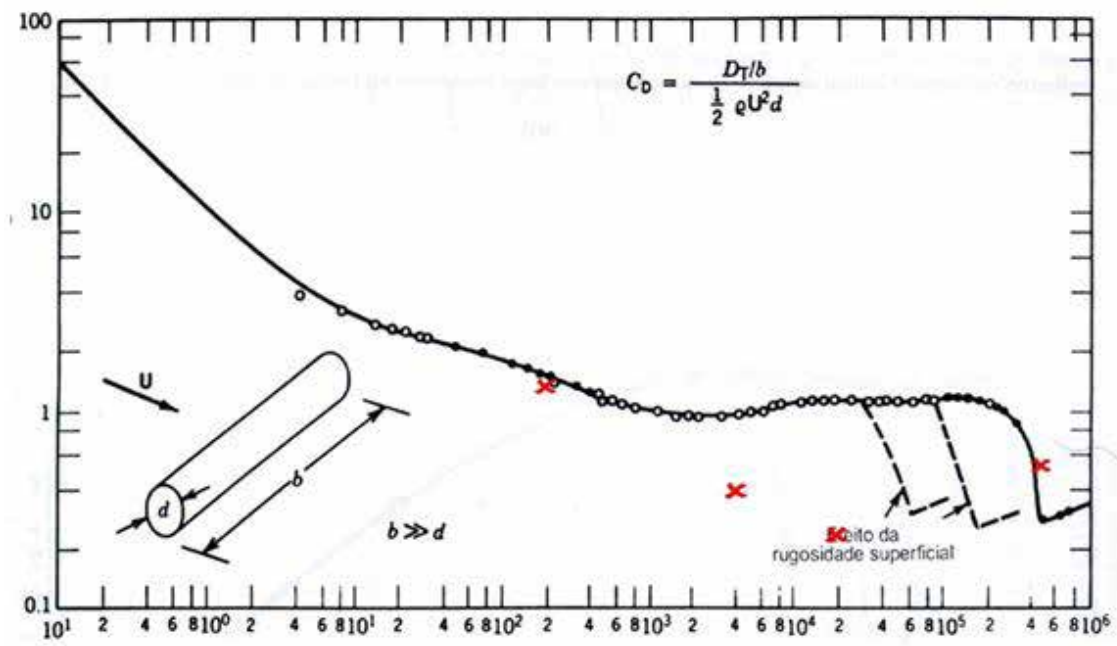

Fonte: Adaptado de SCHMIDT; HENDERSON; WOLGEMUTH (1996) 
Figura 9 - Pontos respectivos aos coeficientes de arrasto obtidos para o modelo $k$ - $\omega$, destacados no gráfico pelo o símbolo $x$

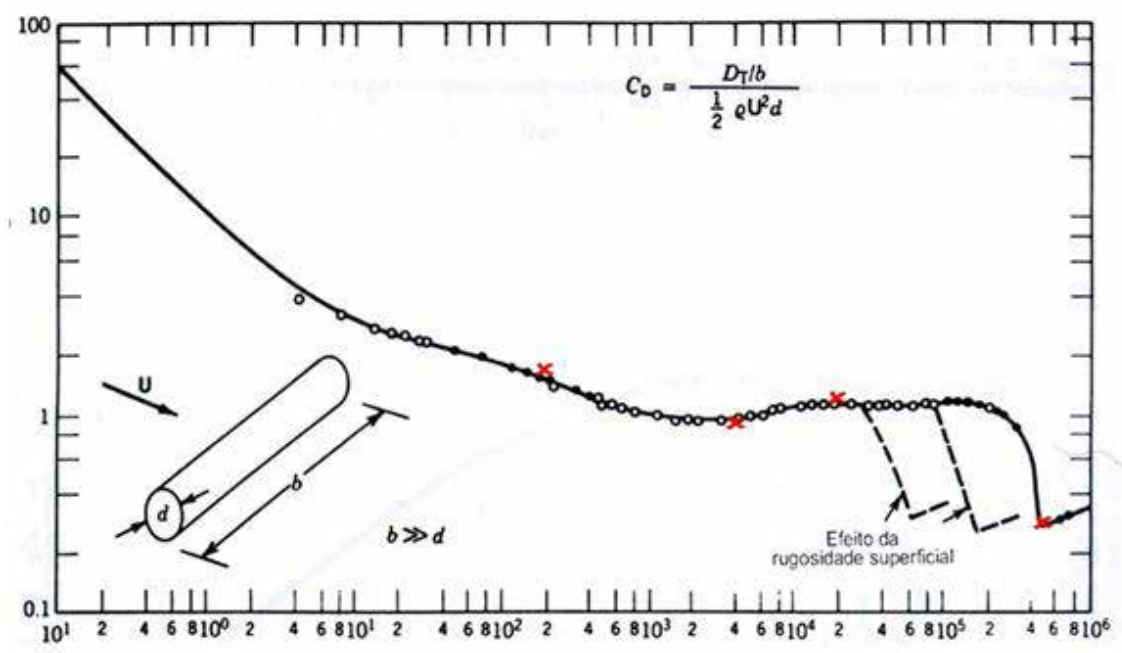

Fonte: Adaptado de SCHMIDT; HENDERSON; WOLGEMUTH (1996)

Pode-se observar, neste caso, que o modelo de turbulência $k$ - $\omega$ apresenta bons resultados para todas as condições de turbulência, desde o início da mesma, prevendo até a chamada crise de arrasto. Sabe-se que este modelo apresenta resultados satisfatórios para escoamentos que possuem gradiente de pressão adverso e para escoamentos recirculantes.

\section{Resultados para o modelo de turbulência SST}

Para o modelo de turbulência SST foram obtidos os resultados apresentados na Tabela VI.
Tabela VI - Resultado do coeficiente de arrasto para o modelo SST

\begin{tabular}{ccccc}
\hline$R e$ & $V[\mathrm{~m} / \mathrm{s}]$ & $C_{D}(S S T)$ & $C_{D}{ }^{*}$ & Erro (\%) \\
\hline 200 & 0,00178 & 1,43197 & 1,625 & $-11,87$ \\
4000 & 0,03572 & 0,98066 & 1,0 & $-1,93$ \\
20000 & 0,17851 & 1,38535 & 1,25 & 10,82 \\
500000 & 4,46289 & 0,42124 & 0,3 & 40,41 \\
\hline
\end{tabular}

A Figura 10 apresenta a localização desses pontos.

Pode-se observar, neste caso, que o modelo de turbulência SST, por combinar as vantagens dos modelos $k$ - $\varepsilon$ e $k-\omega$, apresenta bons resultados para todas as condições de turbulência, com maior discordância na determinação do $C_{D}$ na região respectiva à crise de arrasto. 
Figura 10 - Pontos respectivos aos coeficientes de arrasto obtidos para o modelo SST, destacados no gráfico pelo o símbolo $x$

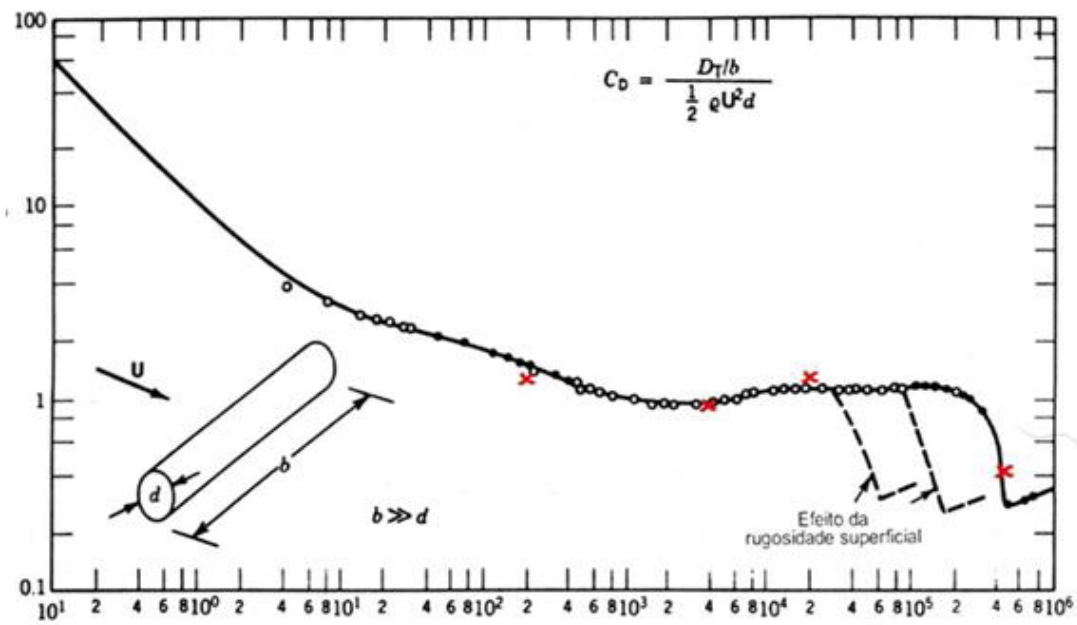

Fonte: Adaptado de SCHMIDT; HENDERSON; WOLGEMUTH (1996)

\section{Comparação dos resultados obtidos para os três modelos de turbulência}

Comparando todos os modelos de turbulência analisados para os pontos escolhidos, pode-se observar que o modelo de turbulência que melhor descreve cada ponto é o modelo $k$ - $\omega$ (Tabela VII).
A Figura 11 representa o comportamento do fluido em torno do cilindro para os quatro pontos escolhidos, onde se aplica o modelo de turbulência $k-\omega$.

Assim, para números de Reynolds a partir de 4000, pode-se observar o surgimento de gradientes de pressão adversos e a presença de zonas de recirculação no escoamento. Esses fenômenos são devidamente tratados pelo modelo de turbulência $k-\omega$.

Tabela VII - Resultado do coeficiente de arrasto para os modelos $k-\varepsilon, k-\omega$ e SST

\begin{tabular}{cccccc}
\hline$R e$ & $V[\mathrm{~m} / \mathrm{s}]$ & $C_{D}(k-\varepsilon)$ & $C_{D}(k-)$ & $C_{D}(\mathrm{SST})$ & $C_{D}{ }^{*}$ \\
\hline 200 & 0,00178516 & 1,49333 & 1,75452 & 1,43197 & 1,625 \\
4000 & 0,0357284 & 0,406861 & 0,957982 & 0,980661 & 1 \\
20000 & 0,178516 & 0,258629 & 1,29358 & 1,38535 & 1.25 \\
500000 & 4,46289 & 0,547248 & 0,332426 & 0,421247 & 0,3 \\
\hline
\end{tabular}


Figura 11 - Linhas de corrente obtidas nos quatro pontos escolhidos para o modelo $k$ -

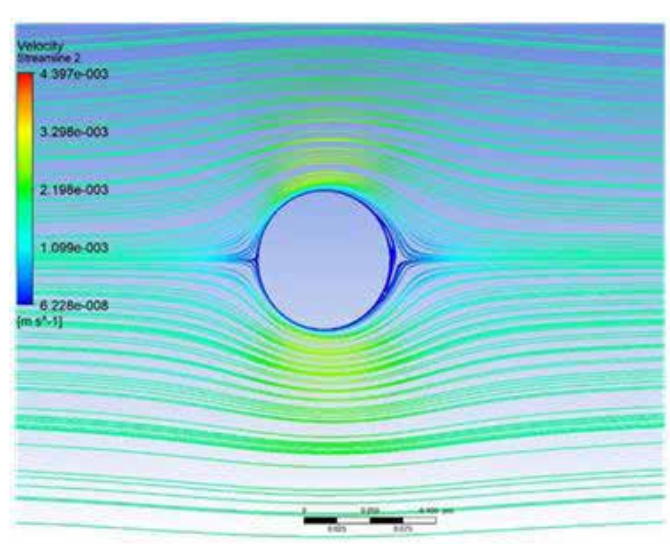

(a) $\operatorname{Re}=200$

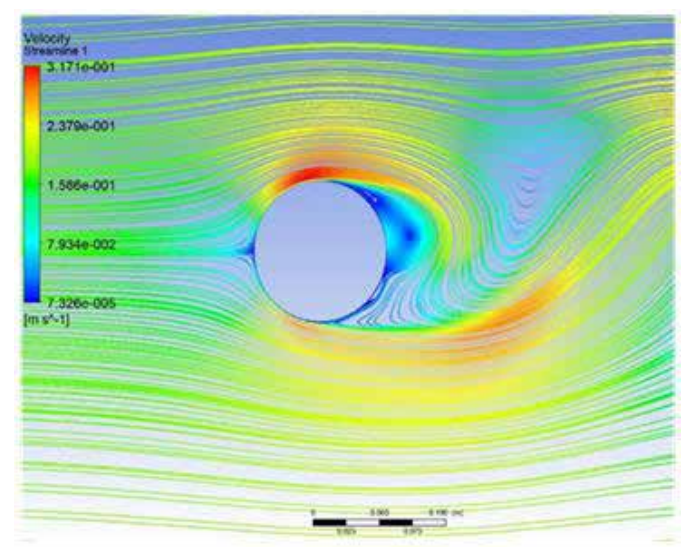

(c) $R e=20000$

\section{Considerações Finais}

O escoamento cruzado de um fluido sobre um cilindro foi simulado por meio do software computacional Ansys CFX, no qual três modelos de turbulência foram analisados: modelos $k-\varepsilon, k-\omega$, e SST. Através das simulações foram obtidos os valores do coeficiente de arrasto para cada modelo, comparando-os aos encontrados na literatura. Com os resultados determinados, foi possível concluir que o modelo de turbulência $k$ - $\varepsilon$ foi

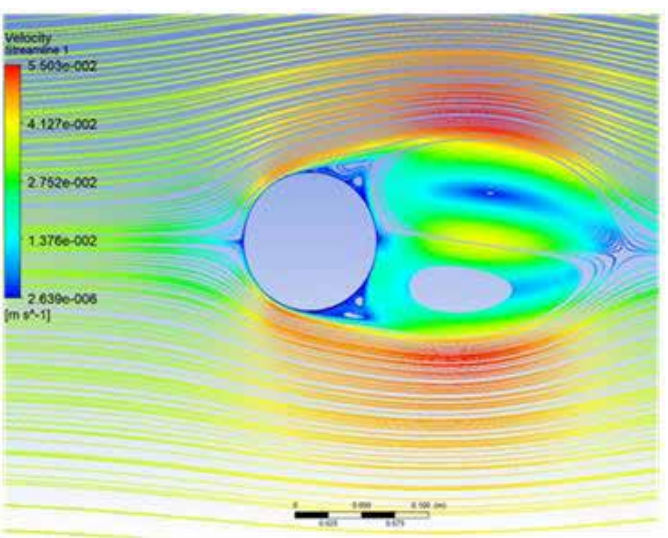

(b) $R e=4000$

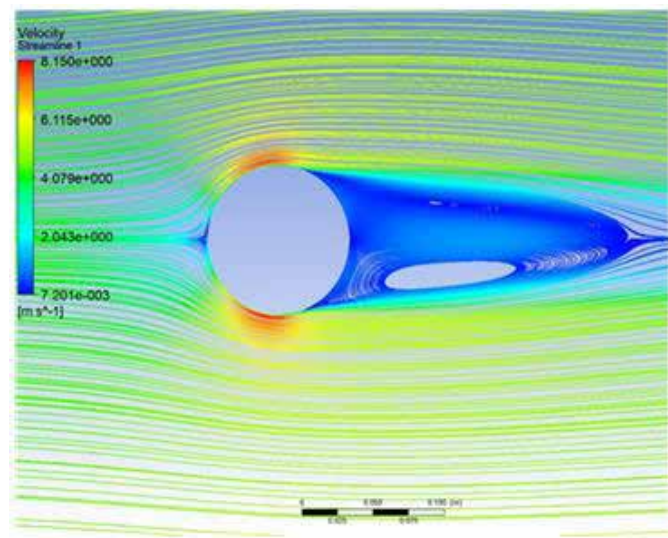

(d) $R e=500000$

o que apresentou os piores resultados, não se mostrando uma boa alternativa para este tipo de escoamento, validando suas características, sendo que possui problemas em alguns casos, como fluxos complexos, separação da camada-limite, camadas-limite curvadas, as quais são características da geometria em estudo. Como observado nos resultados, as simulações para os modelos de turbulência $k$ - $\omega$ e SST mostraram uma boa precisão nos resultados, destacando entre eles o modelo $k-\omega$, que conseguiu prever com excelente precisão uma região crítica do escoamento, a chamada crise de arrasto. Os modelos de 
turbulência $k-\omega$ e SST possuem um comportamento similar para este modelo de geometria, apresentando valores próximos aos encontrados na literatura, apresentando-se como boas alternativas para avaliar esse tipo de escoamento. Porém, o modelo $k$ - $\omega$ é o mais indicado para o escoamento cruzado em cilindro, pois, além de obter bons resultados, ele demanda um menor tempo de simulação em comparação com o modelo SST.

\section{REFERÊNCIAS}

ANSYS CFX Inc., Solver Theory.

BENDER, A. Análise numérica do escoamento turbulento em trocadores de calor com fluxo cruzado: Estudo de um novo arranjo geométrico trapezoidal, 2017. 124 f. Dissertação de Mestrado, Universidade do Estado de Santa Catarina - UDESC, Joinville, SC, 2017.

FOX, R. W.; MCDONALD, A. T. Introdução à mecânica dos fluidos, 8. ed. LTC - Livros Técnicos e Científicos Editora S.A., Rio de Janeiro, RJ, 2014.

MUNSON B.R.; YOUNG D.F.; OKIISHI T. H., Fundamentos da mecânica dos fluidos. $4^{\circ}$ ed. São Paulo. 2004.

PEREIRA, I. A. B. B. Escoamento turbulento em torno de um cilindro a baixo número de Reynolds, 2010. 75 f. Dissertação de Mestrado Integrado em Engenharia Mecânica, Faculdade de Engenharia da Universidade do Porto, 2010.

SCHIMIDT, F. W; HENDERSON, R. E; WOLGEMUTH, C. H., Introdução às ciências térmicas: termodinâmica, mecânica dos fluidos e transferência de calor, 1. ed. ed. Blucher, São Paulo, SP, 1996.

SOARES, D. V. Um modelo $\boldsymbol{k}$ - $\boldsymbol{\omega}$ para escoamentos turbulentos parietais dilatáveis, 2006. 154 f. Dissertação de Mestrado em Ciências Mecânicas, Universidade de Brasília - UnB, Brasília, DF, 2006. 